\title{
Generation of 8QAM-OFDM Optical Access Signals Using DMT Modulation Method
}

\author{
Yufeng Shao ${ }^{1,2, a}$, Wenzhe $\mathrm{Ma}^{1}$, Changxiang $\mathrm{Li}^{1}$, \\ Yue Zhou ${ }^{1}$, Junyi Zhou ${ }^{1}$ and Xingping $\mathrm{Ji}^{1}$ \\ ${ }^{1}$ Faculty of Engineering, Shanghai Polytechnic University, Shanghai, China \\ ${ }^{2}$ Key Laboratory of Signal and Information Processing, College of Electronic and Information \\ Engineering, Chongqing Three Gorges University, Chongqing, China \\ asyufeng@163.com
}

Keywords: optical access system; OFDM; 8QAM; DMT; BER

\begin{abstract}
In this letter, a 8QAM-DMT-OFDM optical access scheme is proposed and demonstrated. 8QAM-OFDM optical access signal is generated using discrete multitone (DMT) modulation, and its optical spectrum, electric spectrum before electro-optical conversion, constellation diagrams before and after transmission and bit error rate (BER) are measured. By this scheme, the system complexity and costs could be reduced.The results show that $\mathrm{t}$ As the transmission distance is $20 \mathrm{~km}$, the value of BER of 8QAM-DMT-OFDM optical access signals is below $10^{-4}$. Therefore, this work not only reduces the complexity and cost budget of 8QAM-DMT-OFDM optical access system but also improves the receiver sensitivity.
\end{abstract}

\section{Introduction}

At present, orthogonal frequency division multiplexing (OFDM) has been widely used in many optical access systems, such as OFDM modulated WDM-PON [1-2], OFDM based metro access [3], and OFDM-ROF systems [4-6]. The reason is that it has a good ability to resist chromatic dispersion (CD) and polarization mode dispersion (PMD), and the spectrum utilization rate is high and the flexible multi-service bandwidth allocation can be realized by using a large number of sub carriers [7-8].OFDM system not only has not guard band between sub channels, but also the main lobe of the spectrum of adjacent channel signals overlaps with each other .But the spectrum of each sub channel signal is orthogonal to each other in the frequency domain, and each subcarrier is orthogonal in time domain. The OFDM sub channel can also use multi-band modulation, and further improve the spectral efficiency of OFDM systems. When the frequency selective fading occurs in the channel due to multipath transmission, only sub carriers that fall in the frequency band and the information they carry are affected. Therefore, the overall BER performance of the system is better. The DMT modulation is a special form of multi-carrier modulation (MCM), which obtains maximum information flow by transmitting different length bits to different sub channels. The output signal after the inverse fast Fourier transform (IFFT) using DMT is real valued signal. In this paper, the downstream transmission of 8QAM-DMT-OFDM optical signals in broadband optical access system has been compared and analyzed. After modulation and coding in this scheme, 8QAM-OFDM optical access signal is downlink transmitted successfully over single mode fiber (SMF) with $2.5 \mathrm{~km}, 5 \mathrm{~km}$, $10 \mathrm{~km}, 15 \mathrm{~km}$ and $20 \mathrm{~km}$ distance at 10Gbit/s.

\section{System Setup}

The DMT is one subclass of OFDM, where the output signals after the inverse fast Fourier transform (IFFT) is real valued signal. If the DMT modulation is introduced in the OOFDM system, in-phase and quadrature (IQ) will not be required. When passband OFDM is utilized, the digital bit stream is converted first into an equivalent baseband signal, and then to a RF signal through 
subcarrier modulation. However, if DMT is utilized, the bandwidth of the generated electrical spectrum will be enlarged to twice range of the original signal, which is equal to the minimum value of the enlarged bandwidth using subcarrier modulation, where the out-put of the IFFT modulation is real instead of complex-valued.

We propose and set up an 8QAM-DMT-OFDM optical access system structure, as shown in Figure 1. At the transmitter side of the downlink, the 10Gbit/s input data is modulated by 8QAM, and then 8QAM electric signals are S/P conversed, DMT modulated, P/S conversed and added cyclic prefix (CP) operation. And then, 10Gbit/s 8QAM-OFDM electrical signals are generated. Through one external cavity laser (ECL), one continuous optical carrier is generated which line width is $100 \mathrm{kHz}$ and the working wavelength is $1550 \mathrm{~nm}$. The generated optical carrier is input to one Mach-Zehnder modulator (MZM). Simultaneously, $10 \mathrm{Gbit} / \mathrm{s}$ 8QAM-OFDM electric signals are input to the MZM for generating 8QAM-OFDM optical signals. When 8QAM-OFDM optical signals are passed through the single mode fiber (SMF), it is amplified by one tunable erbium doped fiber amplifier (EDFA) to compensate attenuation in the optical fiber link. At the receiver, a high sensitivity photo-diode (PIN) is used to detect the $10 \mathrm{Gbit} / \mathrm{s}$ 8QAM-OFDM optical access signal and complete the photoelectric conversion, and the generated 8QAM-OFDM electrical signal is connected to an electric amplifier (EA). The amplified electrical signal is divided into two ways. After the implementation of phase adjustment by one adjustable phase shifter, one is input to the electric mixer for direct mixing with another signal to complete the coherent detection process. A low pass filter (LPF) with the bandwidth of $7.5 \mathrm{GHz}$ is used, and thus $10 \mathrm{Gbit} / \mathrm{s}$ 8QAM-OFDM electrical signals are restored. The detected signal is implemented S/P conversed, removed CP, DMT demodulated, P/S conversed and 8QAM demodulated. At last, $10 \mathrm{Gbit} / \mathrm{s}$ data are restored. Considering the phase sensitivity, the phase matching process must be realized by adjusting the tunable phase shifter, whose dynamic adjustment range is from 0 to 360 degree. By using an optical spectrum analyzer (OSA) in which the $3 \mathrm{~dB}$ resolution bandwidth is $0.02 \mathrm{~nm}$, the optical spectrum of 8QAM-DMT-OFDM optical signals before transmission and the electric spectral diagram of 8QAM-DMT-OFDM baseband signals are shown in Figure 2. The constellation diagrams of 8QAM-DMT-OFDM signals before and after transmission are shown in Figure 3.

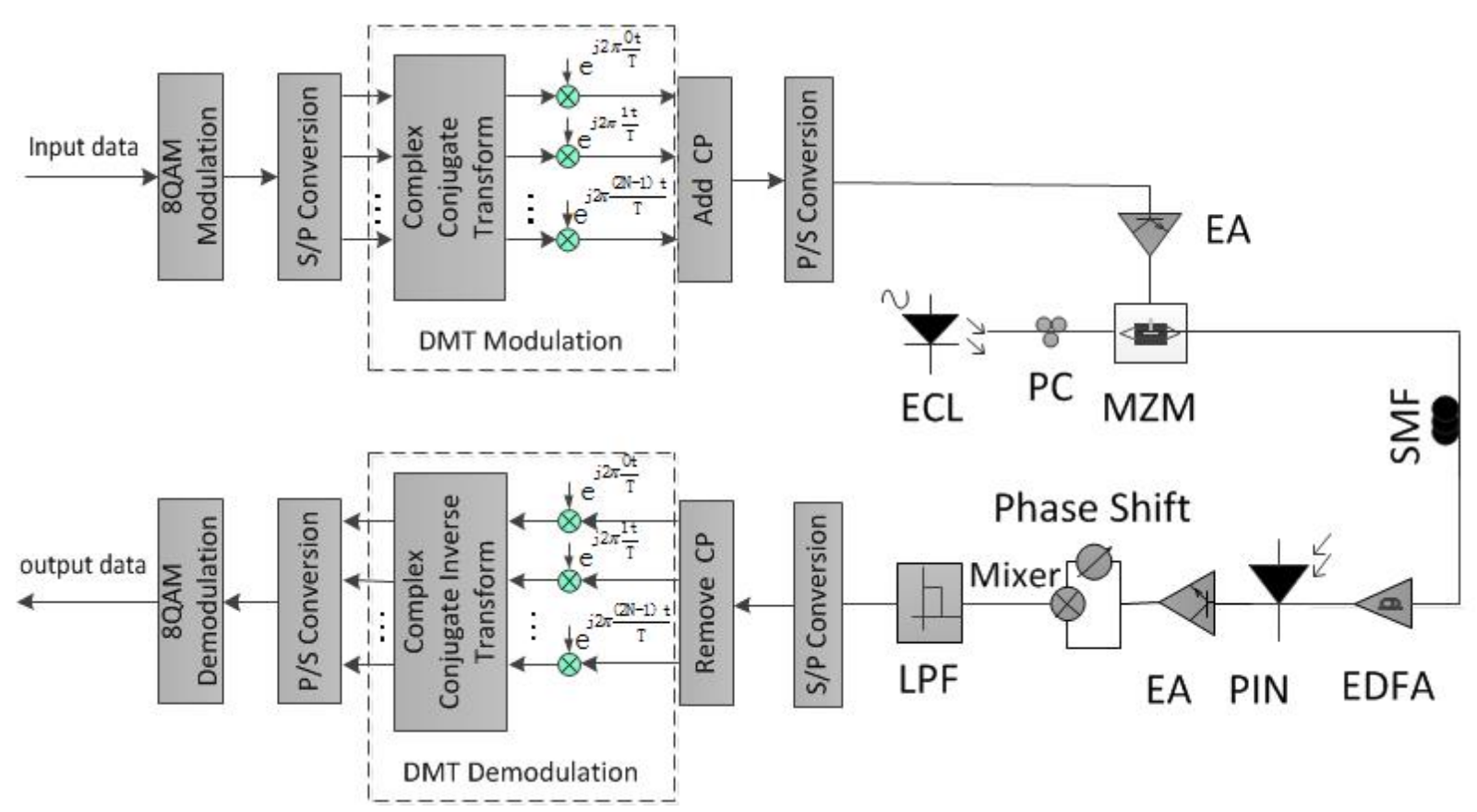

Figure 1. 8QAM-DMT-OFDM optical access system structure

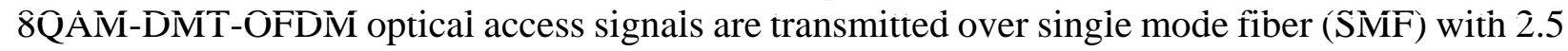
$\mathrm{km}, 5 \mathrm{~km}, 10 \mathrm{~km}, 15 \mathrm{~km}$ and $20 \mathrm{~km}$ distance at 10Gbit/s. The relationship between the bit error rate (BER) and different optical links length is shown in Figure 4.We can find obviously, that if the transmission distance is longer, the values of the BER of the 8QAM-DMT-OFDM optical access 
signals will increase. When the transmission distance is less than $10 \mathrm{~km}$, the BER values of 8QAM-DMT-OFDM optical access signals are lower than $10^{-5}$. As the transmission distance is $20 \mathrm{~km}$, the BER value of 8QAM-DMT-OFDM optical access signals is below $10^{-4}$.
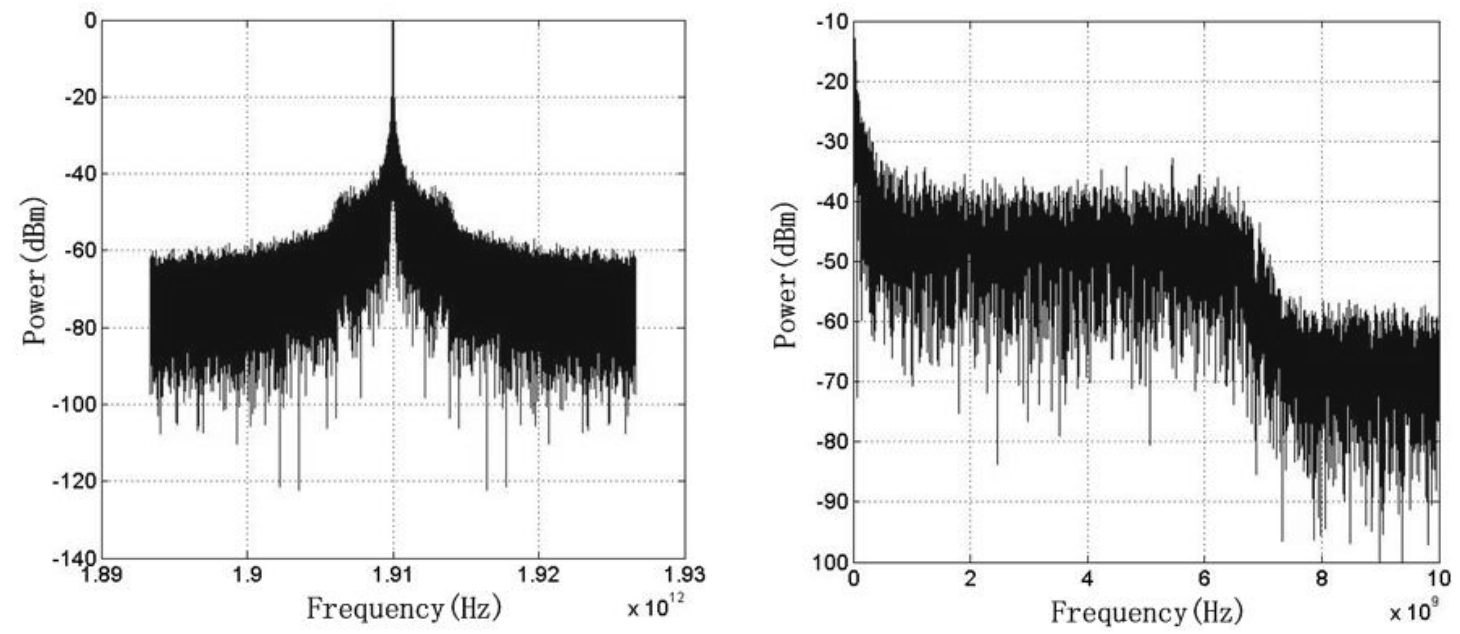

Figure 2. Optical spectral diagram of 8QAM-DMT-OFDM optical access signals and electric spectral diagram of 8QAM-DMT-OFDM baseband signals
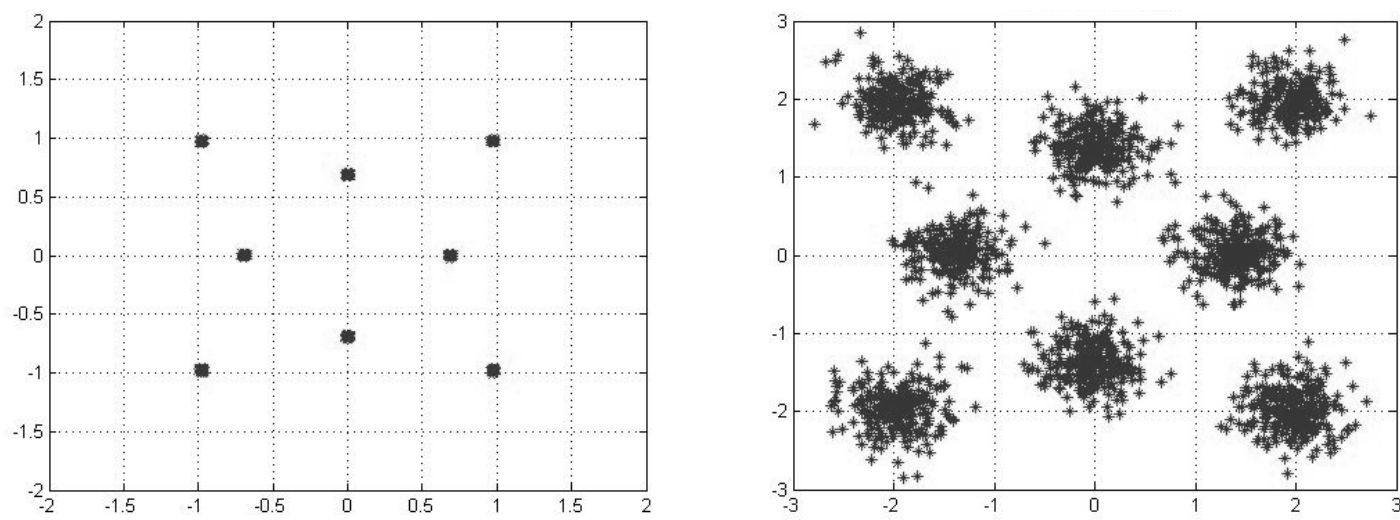

Figure 3. Constellation diagram of 8QAM-DMT-OFDM signals before and after transmission

\section{Summary}

OFDM technology because of the many advantages is applied to the optical access system widely. The DMT reduce OFDM optical access system complexity and costs, while broadband, high-frequency and analog radio frequency (RF) components required for IQ modulation are omitted from transceivers and this design leads to a much simple and cost-effective integrated circuit(IC) design with minimum RF feature. Comparing with 16QAM-OFDM optical access signals, the digital signal processing (DSP) process of 8QAM-OFDM is simpler and with respect to the 16QAM-OFDM optical signals, 8QAM-OFDM optical access signals have a higher frequency band utilization rate. We establish and simulate the 8QAM-DMT-OFDM optical access system. And study the optical spectral diagram of 8QAM-DMT-OFDM optical signals before transmission and the electric spectral diagram of 8QAM-DMT-OFDM baseband signals by using an optical spectrum analyzer (OSA) in which the $3 \mathrm{~dB}$ resolution bandwidth is $0.02 \mathrm{~nm}$. The constellation diagrams of 8QAM-DMT-OFDM signals before and after transmission are measured. The relationship between the bit error rate (BER) and different optical links length is analyzed. The results show that, when the transmission distance is less than $10 \mathrm{~km}$, the BER values of 8QAM-DMT-OFDM optical access signals are lower than $10^{-5}$. As the transmission distance is $20 \mathrm{~km}$, the BER value of 8QAM-DMT-OFDM optical access signals is below $10^{-4}$. Therefore, it is a potential scheme with high reliability in future optical access system. 


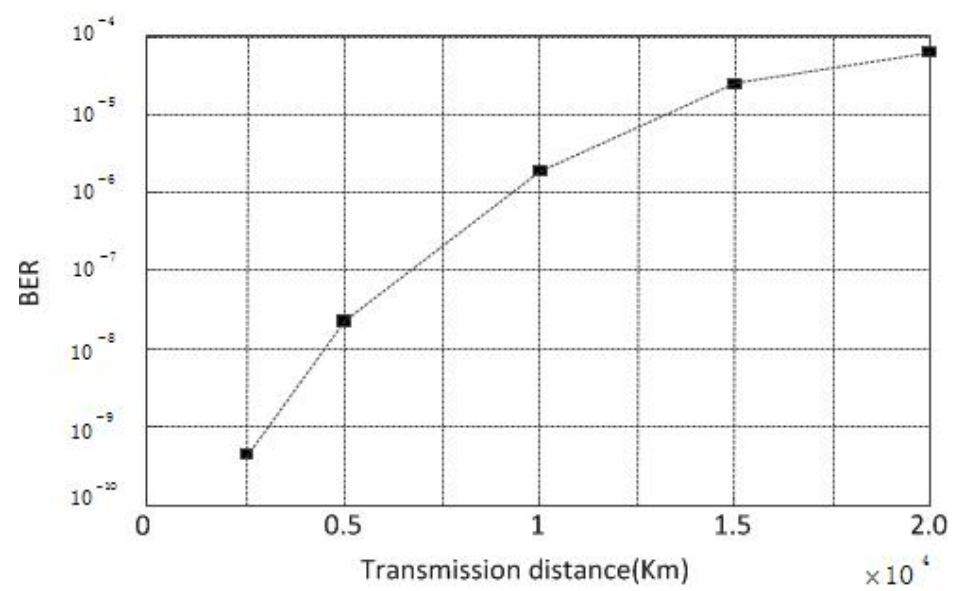

Figure 4. BER of 8QAM-DMT-OFDM optical signal over different length SMF

\section{Acknowledgements}

This work was partially supported by the National Natural Science Foundation of China (No.61107064), Innovation Program of Shanghai Municipal Education Commission (No.15ZZ101), Leading Academic Discipline Project of Information and Communication Engineering (No.XXKZD1605), School Foundation (No.EGD14XQD01) of Shanghai Polytechnic University, College Students' Science and Technology Innovation Project of Shanghai Polytechnic University (No.2016-xjkj-063), Connotation construction project of Shanghai Polytechnic University (No.A11NH170301).

\section{References}

[1] Banerjee A, Park Y, Clarke F, et al. Wavelength-division-multiplexed passive optical network (WDM-PON) technologies for broadband access: a review [Invited]. Journal of Optical Networking, 2005, 4(11):737-758.

[2] Lee W, Park M Y, Cho S H, et al. Bidirectional WDM-PON based on gain-saturated reflective semiconductor optical amplifiers. IEEE Photonics Technology Letters, 2005, 17(11):2460-2462.

[3] Jin W, Zhang C, Chen C, et al. Scalable and reconfigurable all-optical VPN for OFDM-based metro-access integrated, network. Journal of Lightwave Technology, 2014, 32(2):318-325.

[4] Tao L, Yu J, Zhang J, et al. Reduction of intercarrier interference based on window shaping in OFDM rof systems. Photonics Technology Letters IEEE, 2013, 25(9):851-854.

[5] Shao Y, Chi N. PAPR reduction and computational complexity analysis of interleaved segmentation in $60 \mathrm{GHz}$ OFDM-RoF system. Optical Fiber Communication Conference and Exposition and the National Fiber Optic Engineers Conference. 1999:825-832(8).

[6] Shao Y, Ma W, Hu Y, et al. Study on computational complexity in OFDM-RoF system using SLM technique. Fifth International Conference on Instrumentation \& Measurement, Computer, Communication and Control. 2015.

[7] Dardari D, Tralli V, Vaccari A. A theoretical characterization of nonlinear distortion effects in OFDM systems. IEEE Transactions on Communications, 2014, 48(10):1755-1764.

[8] Peng C, Shi Z J, Zhu S J. An overview of peak-to-average power ratio reduction techniques for O-OFDM system. Journal of Anhui Vocational College of Electronics \& Information Technology, 2014. 